\title{
Optimization Solution of Laser Plane Generated from Maximum Likelihood Estimation of Projection Plane
}

\author{
Guan Xu, ${ }^{1}$ Anqi Zheng, ${ }^{1}$ Xiaotao Li, ${ }^{2 *}$ and Jian $\mathrm{Su}^{1}$ \\ ${ }^{1}$ Department of Vehicle Operation Engineering, Traffic and Transportation College, Nanling Campus, \\ Jilin University, Renmin Str. 5988\#, Changchun 130025, China \\ ${ }^{2}$ School of Mechanical Science and Engineering, Nanling Campus, Jilin University, \\ Renmin Str. 5988\#, Changchun 130025, China
}

(Received July 18, 2017; accepted October 4, 2017)

Keywords: active vision, optimization solution, laser plane, MLE

An enhanced solution method that based on the maximum likelihood estimation (MLE) of the projection plane is proposed. The method utilizes a $2 \mathrm{D}$ target and an optimization process to achieve a higher accuracy and noise immunity. The projection plane is parameterized by the laser plane coordinates. The maximum likelihood function is obtained from the stochastic vector of the projection planes in the camera coordinate system. The coordinates of the laser plane are achieved by the optimization process. The MLE method is compared with the original method. The experimental results reveal the relative error reductions of 18.97, 19.81, 21.22, and $21.60 \%$ under the test distances of 200,300, 400, and $500 \mathrm{~mm}$, respectively. Moreover, for the images under noise, the results show the relative error reductions of 20.06, 18.77, 19.64, and $20.35 \%$, respectively. The experimental results suggest that the MLE of the projection plane would be useful to improve the measurement accuracy of active vision in both fundamental research and potential applications.

\section{Introduction}

Laser planes demonstrate remarkable applications in robotic automation, position detection, and profile meters. ${ }^{(1-3)}$ The accurate coordinates of the laser plane are a key point of the measurement precision in active vision. ${ }^{(4,5)}$ Researchers have developed many approaches to obtain the coordinates of the laser plane. On the basis of the dimensions of the target used in the solution process, the solution methods in the active vision are approximately divided into 3D, 2D, and 1D target-based solution methods. ${ }^{(6-10)}$ The 2D planar target is chosen to obtain the laser plane because of the simple operation and abundant information on the target. The applications of the laser plane are being explored by many researchers. Santolaria et al. ${ }^{(11)}$ presented a method for integrating the laser triangulation sensors (LTSs) in the articulated arm of coordinate measuring machines (CMMs). The technique is easily generalizable for LTSs and CMMs. Zhao et al. outlined a methodology that plans the inspection automatically for CMM with a touch trigger probe and a laser scanner. ${ }^{(12)}$ The methodology is suitable for automatic *Corresponding author: e-mail: lixiaotao@jlu.edu.cn http://dx.doi.org/10.18494/SAM.2018.1665 
dimensional measurement. Rahayem et al. conducted comparative research including the popular ellipse-fitting methods from the laser profile-scan sections. ${ }^{(13)}$ The method can be applied in robot-aided welding, automatic inspection, and 3D reconstruction, which require the 3D points to be captured from the surfaces of objects. Ha proposed a calibration structure that has a triangular hole on its plane, which is used for the extrinsic calibration of a camera and the laser range finder. ${ }^{(14)}$ Ma et al. developed a large-scale calibration system of the laser plane. ${ }^{(15)}$ It consists of a $35 \mathrm{~m}$ horizontal guide rail and a $1.5 \mathrm{~m}$ vertical guide rail. The laser plane can be utilized for a lab standard to calibrate the portable CMMs. Fischer et al. described a method for obtaining the volumetric data with a single frame using a high-speed light-field camera. ${ }^{(16)}$ The high-speed light-field camera approach is used in a Doppler global velocimeter with the sinusoidal laser frequency modulation. Aymerich et al. presented a laser-based technique combined with the Talbot effect for microstructuring surfaces. ${ }^{(17)}$ These results reveal the advantages of the technique for repeatable, rapid, and no-contaminant multistructuring. Chiu et al. proposed a technique for obtaining the surface profile in real time by common-path heterodyne interferometry with a laser to scan the specimen and measure the phase difference distribution. ${ }^{(18)}$ Chen et al. designed a compact $2 \mathrm{D}$ single-mirror laser scanner. ${ }^{(19)}$ The laser scanner is controlled by three piezoelectric actuators aligned in parallel and has high accuracy. Xu et al. employed a 2D target and solved the laser plane by the Plücker matrices of the projection laser lines. ${ }^{(20)}$ Another method employing a 3D target and the distance between the global origin and the point on the laser plane is also reported in the previous work. ${ }^{(21)}$ The existing 2D target solution methods utilize the cross ratio invariability or Plücker matrix to construct the coordinates of a laser plane. ${ }^{(22)}$ As the Plücker matrix directly presents the 3D projection laser line on the target, the measurement technique with the Plücker matrix is considered to be the better method to obtain the laser plane.

As the 2D target is convenient to be manufactured, the 2D target is employed in this method. The original method of the Plücker matrix has the advantages of simplicity and high efficiency. However, the recognized laser lines in the images are impacted by noise. Therefore, it should be smoothed by the statistical and optimizing processes. The advantage of the method is that it can solve the laser plane by the maximum likelihood estimation (MLE), which improves the accuracy of the laser plane. First, we construct the solution model based on the projection plane instead of the laser plane. Then, the MLE is employed on the joint Gaussian distribution of the parameterized projection plane to contribute the logarithmic likelihood function. Finally, the solution of the laser plane is enhanced by optimizing the logarithmic likelihood function theoretically and experimentally.

\section{Solution Model}

The projection system consists of a target, a laser projector, and a camera, as shown in Fig. 1. The planar target is located in the view field of the camera. The laser plane of the projector and the target intersect at a line on the target. The images of the target and the projection line are captured by the camera. The laser plane is determined by the laser projector. The world, the camera, and the image coordinate systems are defined on the target, the camera, and the image, respectively. 


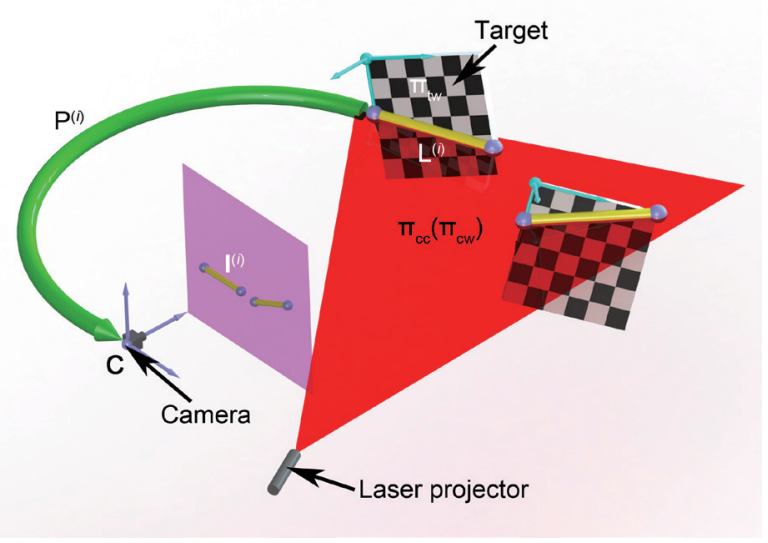

Fig. 1. (Color online) Solution model of the laser plane using MLE.

As illustrated above, in the world coordinate system, the projection plane $\pi_{c w}^{(i)}$ passing through the camera center $\boldsymbol{C}$ and the 2D projection line $\boldsymbol{l}^{(i)}$ in the $i$ th image is determined by ${ }^{(20)}$

$$
\boldsymbol{\pi}_{c w}^{(i)}=\left(\boldsymbol{P}^{(i)}\right)^{\mathrm{T}} \boldsymbol{l}^{(i)}
$$

where $\boldsymbol{P}^{(i)}$ is the projection matrix of the camera and can be generated from the camera calibration. ${ }^{(23)}$

In the camera coordinate system, the estimated projection plane $\pi_{c w}^{(i)}$ is transformed to ${ }^{(20)}$

$$
\boldsymbol{\pi}_{c c}^{(i)}=\left(\boldsymbol{H}^{(i)}\right)^{-\mathrm{T}}\left(\boldsymbol{P}^{(i)}\right)^{\mathrm{T}} \boldsymbol{l}^{(i)}
$$

where $\boldsymbol{H}^{(i)}$ is the calibrated transform matrix of the $i$ th image from the world coordinate system to the image coordinate system. ${ }^{(20)}$

In Fig. 1 , the $3 \mathrm{D}$ intersection line is derived from the target plane $\boldsymbol{\pi}_{t w}=[0,0,1,0]^{\mathrm{T}}$ in the world coordinate system and the parameterized laser plane $\pi_{p}(a, b, c, d)$ in the camera coordinate system. It can be solved as

$$
\boldsymbol{L}^{(i)}=\boldsymbol{\pi}_{p}(a, b, c, d)\left[\left(\boldsymbol{H}^{(i)}\right)^{-\mathrm{T}} \boldsymbol{\pi}_{t w}^{(i)}\right]^{\mathrm{T}}-\left[\left(\boldsymbol{H}^{(i)}\right)^{-\mathrm{T}} \boldsymbol{\pi}_{t w}^{(i)}\right]\left[\boldsymbol{\pi}_{p}(a, b, c, d)\right]^{\mathrm{T}},
$$

where $a, b, c$, and $d$ are the coordinates of the laser plane.

In another way, the accurate projection plane $\pi_{c c}^{(i)}$ is denoted in the camera coordinate system by

$$
\overline{\boldsymbol{\pi}}_{c c}^{(i)}=\boldsymbol{L}^{(i)} \boldsymbol{C}
$$

where $\boldsymbol{C}=[0,0,0,1]^{\mathrm{T}}$ is the camera center. 
Substituting Eq. (3) into Eq. (4), the accurate projection plane is parameterized by

$$
\overline{\boldsymbol{\pi}}_{c c}^{(i)}=\left\{\boldsymbol{\pi}_{p}(a, b, c, d)\left[\left(\boldsymbol{H}^{(i)}\right)^{-\mathrm{T}} \boldsymbol{\pi}_{t w}^{(i)}\right]^{\mathrm{T}}-\left[\left(\boldsymbol{H}^{(i)}\right)^{-\mathrm{T}} \boldsymbol{\pi}_{t w}^{(i)}\right]\left[\boldsymbol{\pi}_{p}(a, b, c, d)\right]^{\mathrm{T}}\right\} \boldsymbol{C} .
$$

In the test, a projection plane can be considered as a stochastic variable. We assume that the projection plane $\pi_{c c}^{(i)}$ obeys the Gaussian distribution. Then, the stochastic vector $\boldsymbol{\Pi}_{c}=\left(\boldsymbol{\pi}_{c c}^{(1)}, \boldsymbol{\pi}_{c c}^{(2)}, \ldots, \boldsymbol{\pi}_{c c}^{(n)}\right)^{\mathrm{T}}$ obeys a joint Gaussian distribution as

$$
P\left(\boldsymbol{\Pi}_{c}\right)=(2 \pi)^{-n / 2} \operatorname{det}\left(\Sigma^{-1}\right)^{1 / 2} \exp \left(-\left(\boldsymbol{\Pi}_{c}-\overline{\boldsymbol{\Pi}}_{c}\right)^{\mathrm{T}} \boldsymbol{\Sigma}^{-1}\left(\boldsymbol{\Pi}_{c}-\overline{\boldsymbol{\Pi}}_{c}\right) / 2\right),
$$

where $\boldsymbol{\Sigma}=E\left[\left(\boldsymbol{\Pi}_{c}-\overline{\boldsymbol{\Pi}}_{c}\right)\left(\boldsymbol{\Pi}_{c}-\overline{\boldsymbol{\Pi}}_{c}\right)^{\mathrm{T}}\right]$ is the covariance matrix $\overline{\boldsymbol{\Pi}}_{c}=\left(\overline{\boldsymbol{\pi}}_{c c}^{(1)}, \overline{\boldsymbol{\pi}}_{c c}^{(2)}, \ldots, \overline{\boldsymbol{\pi}}_{c c}^{(n)}\right)^{\mathrm{T}}$.

As the projection planes are obtained by the same independent procedures, $\Sigma=\sigma^{2} \boldsymbol{I} . \sigma^{2}$ is the variance. $\boldsymbol{I}$ is the identical matrix. The joint Gaussian distribution is

$$
P\left(\boldsymbol{\Pi}_{c}\right)=\prod_{i=1}^{n}(\sqrt{2 \pi} \sigma)^{-1} \exp \left(-\left(\boldsymbol{\pi}_{c c}^{(i)}-\overline{\boldsymbol{\pi}}_{c c}^{(i)}\right)^{2} / 2 \sigma^{2}\right)
$$

The logarithmic likelihood function is

$$
\log \left\{P\left(\boldsymbol{\Pi}_{c}\right)\right\}=-\frac{1}{2 \sigma^{2}} \sum_{i=1}^{n}\left(\boldsymbol{\pi}_{c c}^{(i)}-\overline{\boldsymbol{\pi}}_{c c}^{(i)}\right)^{2}-\sum_{i=1}^{n} \log (\sqrt{2 \pi} \sigma) .
$$

Then, the MLE of Eq. (8) is

$$
\min \sum_{i=1}^{n}\left\|\pi_{c c}^{(i)}-\bar{\pi}_{c c}^{(i)}(a, b, c, d)\right\|^{2}
$$

The optimized coordinates of the laser plane in Eq. (9) can be solved by the LevenbergMarquardt (LM) algorithm. ${ }^{(24,25)}$

\section{Experimental Results and Analysis}

A camera with $512 \times 384$ image resolution and a $160 \times 160 \mathrm{~mm}^{2}$ target with a $10 \times 10 \mathrm{~mm}^{2}$ checkerboard pattern are employed to examine the performance of the proposed MLE method. The optimization method is compared with the original method in the two experimental approaches, the reprojection laser lines on the image, and the quantitative errors of reprojection lines. ${ }^{(20)}$

The optimized laser plane and the laser plane of the original method are reprojected to the captured images. Four groups of experiments are performed to verify the measurement accuracy. Fifteen laser lines are extracted from the captured target images in every group of experiments. The reprojection lines of the laser planes in the case of the optimization method and the original method are shown in Fig. 2. In the first group of experiments, the images are 


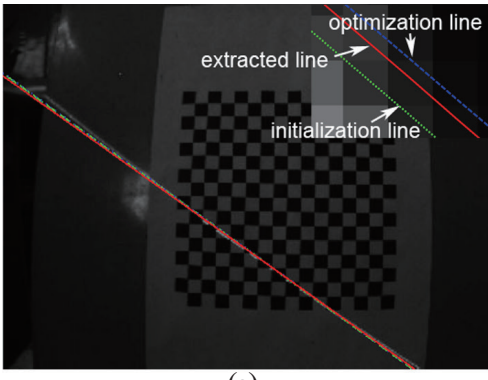

(a)

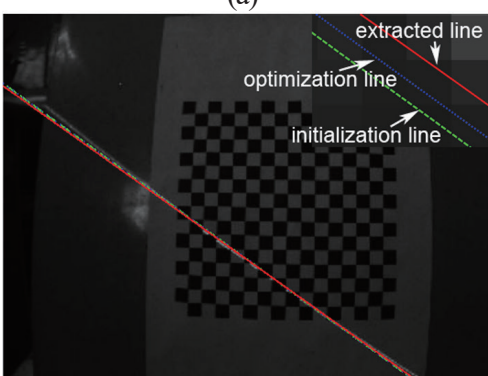

(c)

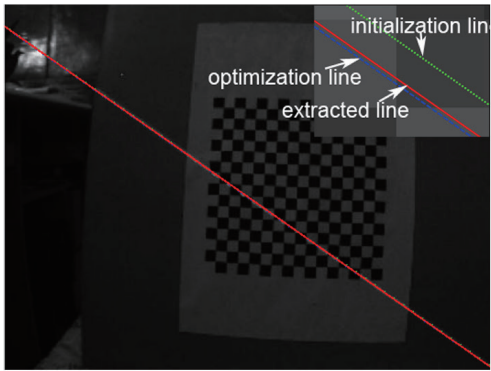

(e)

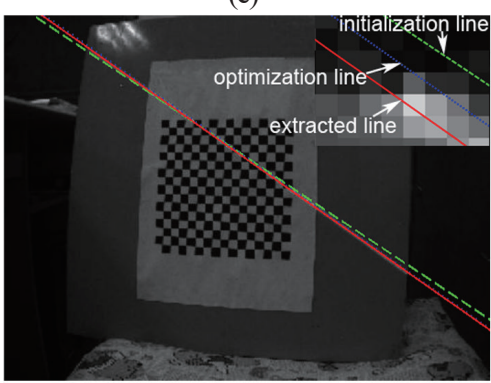

(g)

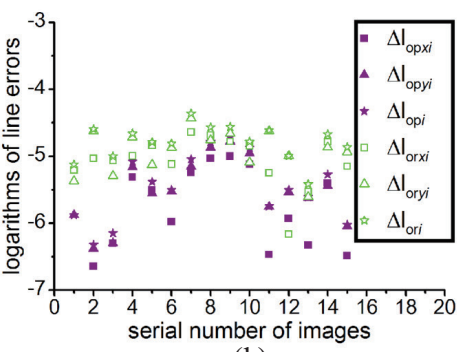

(b)

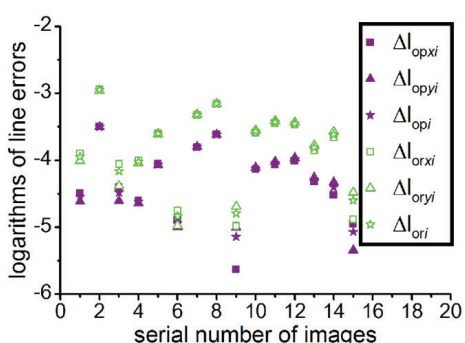

(d)

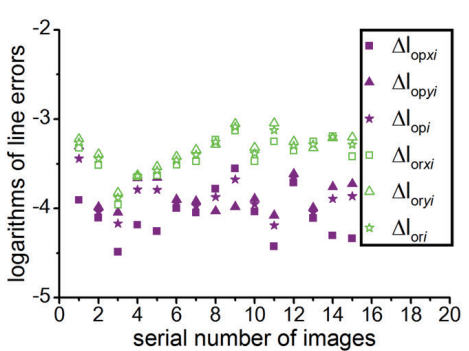

(f)

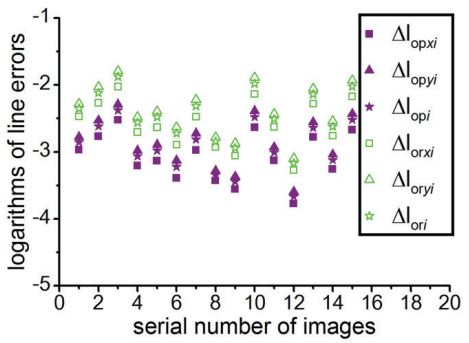

(h)

Fig. 2. (Color online) Reprojection of the MLE solution method and the original method. (a), (c), (e), (g) Projection lines and extracted lines in four groups of experiments. (b), (d), (f), (h) Line reprojection errors in four groups of experiments. $\Delta \boldsymbol{l}_{\text {opxi }}, \Delta \boldsymbol{l}_{\text {opyi }}$, and $\Delta \boldsymbol{l}_{\text {opi }}$ are the $x$ direction, the $y$ direction, and the RMS errors of the MLE method. $\Delta \boldsymbol{l}_{\text {orxi }}, \Delta \boldsymbol{l}_{\text {oryi }}$, and $\Delta \boldsymbol{l}_{\text {ori }}$ are the $x$ direction, the $y$ direction, and the RMS errors of the original method.

captured at the test distance of $200 \mathrm{~mm}$. The solid line is the real laser line, which is considered as the benchmark of the comparison and is generated from the feature extraction. The dashed line of the optimization method is closer to the solid line than the dotted line of the original method. It shows that the proposed MLE method provides a higher precision than the original method. In order to quantize the results, Fig. 2(b) illustrates the logarithmic errors. The mean line errors in the $x$ direction for the optimization method and the original method are $3.25 \times$ $10^{-6}$ and $1.09 \times 10^{-5}$, respectively. The mean line errors in the $y$ direction for the optimization 
method and the original method are $4.97 \times 10^{-6}$ and $1.46 \times 10^{-5}$, respectively. Finally, the root mean squares (RMSs) defined by Eq. $(10)^{(26)}$ of the errors are $6.01 \times 10^{-6}$ and $1.89 \times 10^{-5}$, respectively.

$$
R=\left[(1 / 2)\left(l_{x}^{2}+l_{y}^{2}\right)\right]^{1 / 2},
$$

where $R$ is the RMS, $l_{x}$ and $l_{y}$ are the mean line errors in the $x$ direction and the $y$ direction, respectively. The data indicate that the optimization method contributes the higher accuracy.

In the second group of experiments, the images are captured at the test distance of $300 \mathrm{~mm}$. In Fig. 2(c), the optimization line is closer to the extracted line than the line from the original method. The results show that the optimization method achieves higher accuracy. Meanwhile, the mean line errors in the $x$ direction for the optimization method and the original method are 8.42 $\times 10^{-5}$ and $2.83 \times 10^{-4}$, respectively. The mean line errors in the $y$ direction for the optimization method and the original method are $8.52 \times 10^{-5}$ and $2.86 \times 10^{-4}$, respectively. The RMS of the errors are $8.50 \times 10^{-5}$ and $2.85 \times 10^{-4}$, respectively. For the test distance of $400 \mathrm{~mm}$, similar results can be seen in Fig. 2(e). The optimization line is obviously closer to the extracted line than the initialization line. The mean line errors in the $x$ direction for the optimization method and the original method are $9.92 \times 10^{-5}$ and $4.06 \times 10^{-4}$, respectively. The mean line errors in the $y$ direction for the optimization method and the original method are $1.66 \times 10^{-4}$ and $5.06 \times$ $10^{-4}$, respectively. The RMS of the errors are $1.44 \times 10^{-4}$ and $4.61 \times 10^{-4}$, respectively. Finally, when the test distance is $500 \mathrm{~mm}$, the mean line errors in the $x$ direction for the optimization method and the original method are $1.12 \times 10^{-3}$ and $3.54 \times 10^{-3}$, respectively. The mean line errors in the $y$ direction for the optimization method and the original method are $1.91 \times 10^{-3}$ and $6.03 \times 10^{-3}$, respectively. The RMS of the errors are $1.56 \times 10^{-3}$ and $4.94 \times 10^{-3}$, respectively. The data above reveals that the optimization method shows higher accuracy than the original method. Moreover, the mean line errors in the $x$ direction and $y$ direction, and the RMS errors for the optimization method and original method both increase with increasing test distance.

Furthermore, four levels of Gaussian noise are added to the images to explore the impact of such noise. The noise levels are $0.001,0.005,0.01$, and 0.05 . At a distance of $200 \mathrm{~mm}$, the line logarithmic errors in the $x$ direction and the $y$ direction with noise for the two methods are shown in the Figs. 3(a) and 3(b). In the $x$ direction, the mean errors when using the optimization

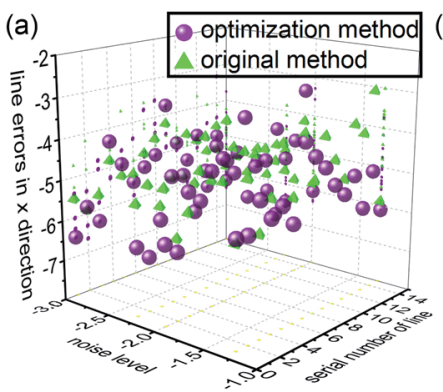

(a)

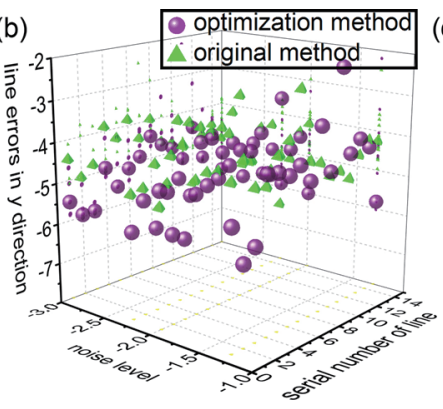

(b)

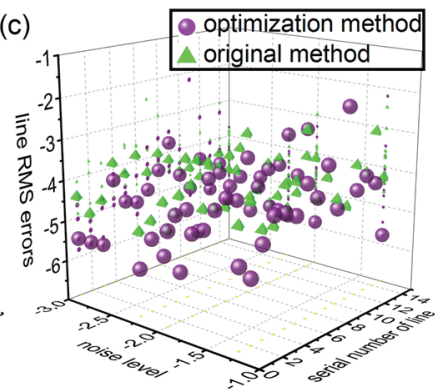

(c)

Fig. 3. (Color online) Line errors of the MLE and the original methods in the first group of experiments with noises. 
method are $1.35 \times 10^{-5}, 1.07 \times 10^{-5}, 6.44 \times 10^{-5}$, and $9.38 \times 10^{-5}$, respectively. The mean errors of the original method are $2.01 \times 10^{-5}, 2.39 \times 10^{-5}, 8.23 \times 10^{-5}$, and $1.36 \times 10^{-4}$, respectively. In the $y$ direction, the mean errors for the optimization method are $1.14 \times 10^{-5}, 1.91 \times 10^{-5}$, $5.81 \times 10^{-5}$, and $9.77 \times 10^{-5}$, respectively. The mean errors in the $y$ direction when using the original method are $4.17 \times 10^{-5}, 8.69 \times 10^{-5}, 1.23 \times 10^{-4}$, and $2.88 \times 10^{-4}$, respectively. The RMS logarithmic errors of the two methods are described in Fig. 3(c). The variation range of the errors when using the optimization method under noise is $1.77 \times 10^{-5}-1.35 \times 10^{-4}$. The variation scope of the errors when using the original method under noise is $4.63 \times 10^{-5}-3.04 \times$ $10^{-4}$.

As the test distance is $300 \mathrm{~mm}$, the line errors with noise in the $x$ direction and the $y$ direction are shown in Figs. 4(a) and 4(b). In the $x$ direction, the mean errors in the case of the optimization method are $1.13 \times 10^{-4}, 1.30 \times 10^{-4}, 2.01 \times 10^{-4}$, and $7.41 \times 10^{-4}$, respectively. The mean errors of the original method are $3.27 \times 10^{-4}, 4.75 \times 10^{-4}, 1.17 \times 10^{-3}$, and $1.44 \times 10^{-3}$, respectively. In the $y$ direction, the mean errors for the optimization method are $5.16 \times 10^{-5}$, $1.37 \times 10^{-4}, 8.05 \times 10^{-4}$, and $6.97 \times 10^{-4}$, respectively. The mean errors in the $y$ direction when using the original method are $8.65 \times 10^{-4}, 1.47 \times 10^{-3}, 1.63 \times 10^{-3}$, and $1.06 \times 10^{-3}$, respectively. Figure 4(c) shows the RMS errors of the two methods. The variation range of the errors when using the optimization method with noise is $9.17 \times 10^{-5}-8.15 \times 10^{-4}$. The variation scope of the errors when using the original method with noise is $6.71 \times 10^{-4}-1.73 \times 10^{-3}$.

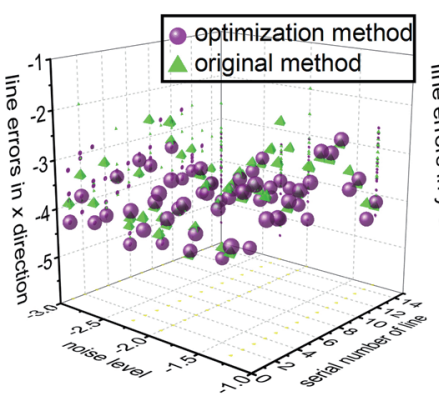

(a)

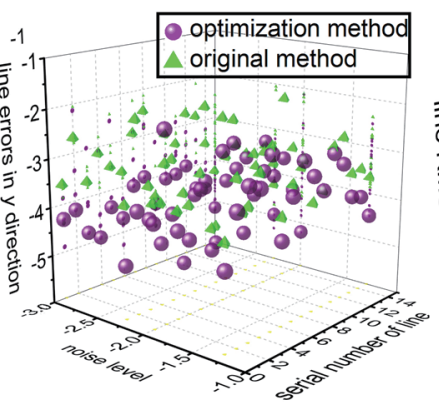

(b)

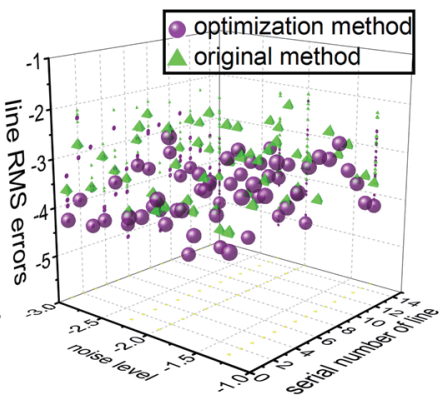

(c)

Fig. 4. (Color online) Line errors of the MLE and the original methods in the second group of experiments with noise.

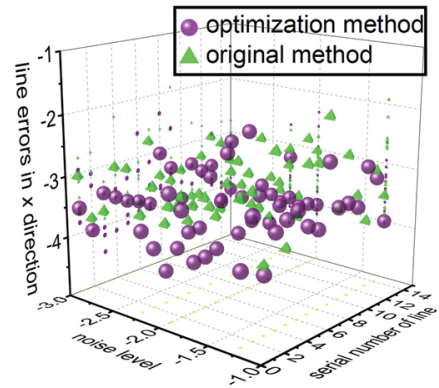

(a)

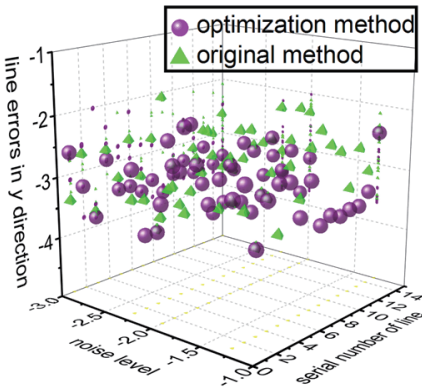

(b)

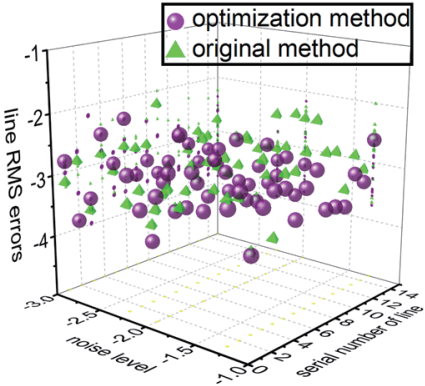

(c)

Fig. 5. (Color online) Line errors of the MLE and the original methods in the third group of experiments with noise. 
For the test distance increasing to $400 \mathrm{~mm}$, the line errors in the two methods are described in Figs. 5(a) and 5(b). In the $x$ direction, the mean errors when using the optimization method are $2.68 \times 10^{-4}, 3.47 \times 10^{-4}, 6.89 \times 10^{-4}$, and $5.44 \times 10^{-4}$, respectively. The mean errors of the original method are $5.35 \times 10^{-4}, 4.68 \times 10^{-4}, 7.16 \times 10^{-4}$, and $2.11 \times 10^{-3}$, respectively. In the $y$ direction, the mean errors for the optimization method are $5.24 \times 10^{-4}, 1.33 \times 10^{-3}, 1.65 \times$ $10^{-3}$, and $1.06 \times 10^{-3}$, respectively. The mean errors in the $y$ direction when using the original method are $1.65 \times 10^{-3}, 1.87 \times 10^{-3}, 2.23 \times 10^{-3}$, and $2.95 \times 10^{-3}$, respectively. The RMS errors in the two methods are described in Fig. 5(c). The variation range of the errors when using the optimization method with noise is $5.25 \times 10^{-4}-1.50 \times 10^{-3}$. The variation scope of the errors when using the original method with noise is $7.14 \times 10^{-4}-2.40 \times 10^{-3}$.

For increasing test distance to $400 \mathrm{~mm}$, the line errors when using the two methods are described in Figs. 5(a) and 5(b). In the $x$ direction, the mean errors when using the optimization method are $2.68 \times 10^{-4}, 3.47 \times 10^{-4}, 6.89 \times 10^{-4}$, and $5.44 \times 10^{-4}$, respectively. The mean errors of the original method are $5.35 \times 10^{-4}, 4.68 \times 10^{-4}, 7.16 \times 10^{-4}$, and $2.11 \times 10^{-3}$, respectively. In the $y$ direction, the mean errors for the optimization method are $5.24 \times 10^{-4}, 1.33 \times 10^{-3}$, $1.65 \times 10^{-3}$, and $1.06 \times 10^{-3}$, respectively. The mean errors in the $y$ direction using the original method are $1.65 \times 10^{-3}, 1.87 \times 10^{-3}, 2.23 \times 10^{-3}$, and $2.95 \times 10^{-3}$, respectively. The RMS errors of the two methods are described in Fig. 5(c). The variation range of the errors when using the optimization method with noise is $5.25 \times 10^{-4}-1.50 \times 10^{-3}$. The variation scope of the errors when using the original method with noise is $7.14 \times 10^{-4}-2.40 \times 10^{-3}$.

Finally, the line errors of the two methods with the test distance of $500 \mathrm{~mm}$ are shown in Figs. 6(a) and 6(b). In the $x$ direction, the mean errors when using the optimization method are $2.74 \times 10^{-3}, 3.80 \times 10^{-3}, 1.04 \times 10^{-2}$, and $1.11 \times 10^{-2}$, respectively. The mean errors for the original method are $5.03 \times 10^{-3}, 4.95 \times 10^{-3}, 1.16 \times 10^{-2}$, and $2.48 \times 10^{-2}$, respectively. In the $y$ direction, the mean errors for the optimization method are $2.80 \times 10^{-3}, 4.60 \times 10^{-3}, 7.16 \times$ $10^{-3}$, and $3.14 \times 10^{-2}$, respectively. The mean errors in the $y$ direction when using the original method are $5.13 \times 10^{-3}, 5.50 \times 10^{-3}, 2.85 \times 10^{-2}$, and $3.92 \times 10^{-2}$, respectively. The RMS errors of the two methods are described in Fig. 6(c). The variation range of the errors when using the optimization method with noise is $2.96 \times 10^{-3}-3.13 \times 10^{-2}$. The variation scope of the errors when using the original method with noise is $5.53 \times 10^{-3}-3.24 \times 10^{-2}$. The errors for the two

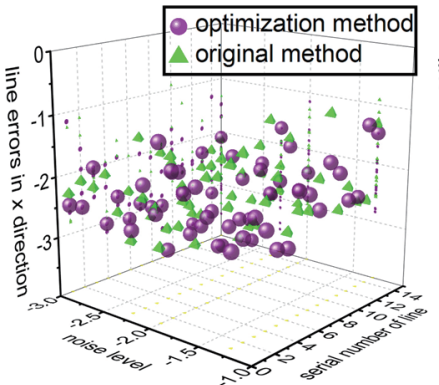

(a)

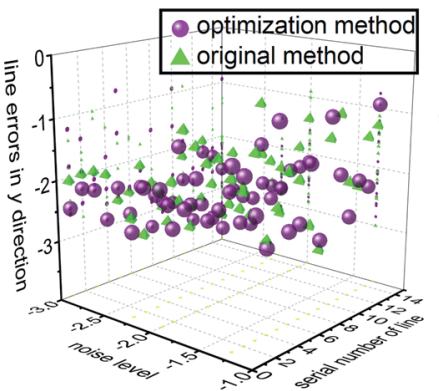

(b)

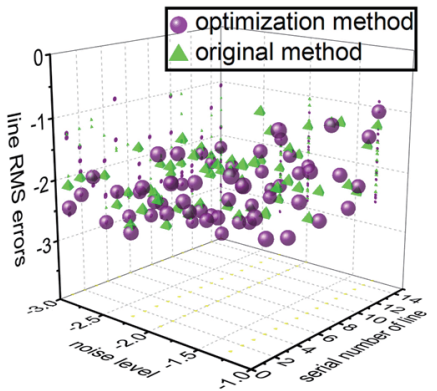

(c)

Fig. 6. (Color online) Line errors of the MLE and the original methods in the fourth group of experiments with noise. 
methods at the different test distances basically increase with increasing noise. Moreover, the errors of the optimization method are all smaller than those of the original method. At the same time, the line errors in the $x$ direction and the $y$ direction basically increase as the test distance increases. The experimental results show that the optimization method provides higher noise immunity.

\section{Conclusion}

An approach for solving a laser plane was proposed in this article. The MLE method was employed to obtain the optimization function of the laser plane. The solution method is based on the Plücker matrix that directly presents the $3 \mathrm{D}$ projection laser line on the target. Four groups of experiments were performed to investigate the measurement accuracy and the noise immunity. The absolute measurement errors of the MLE method were less than the errors of the original method. Similar results were observed in the experiments of testing the influence of noise. Moreover, the experimental results indicated the relative error reductions of 18.97, 19.81, 21.22 , and $21.60 \%$ in the original images and the relative error reductions of $20.06,18.77,19.64$, and $20.35 \%$ in the images with noise. The comparison results revealed that the optimization method presents higher accuracy and noise immunity. The MLE of the projection plane would be beneficial for the applications of profile measurement, vehicle navigation, etc. The future work will focus on the high accuracy solution of the laser plane in a multicamera system.

\section{Acknowledgments}

This work was supported by the National Natural Science Foundation of China (NSFC) (51478204, 51205164) and Natural Science Foundation of Jilin Province (20170101214JC, 20150101027JC).

\section{References}

1 A. S. Poulin-Girard, S. Thibault, and D. Laurendeau: Opt. Express 24 (2016) 2678.

J. Jung, I. Yoon, S. Lee, and J. Paik: J. Sens. 2016 (2016) 1.

A. Glowacz and Z. Glowacz: Measurement 93 (2016) 86.

4 J. Santolaria, J. Conte, and M. Ginés: Int. J. Adv. Manuf. Tech. 66 (2013) 2087.

B. Özgürün, D. Ö. Tayyar, K. Ö. Agiş, and M. Özcan: Appl. Opt. 56 (2017) F84.

6 Y. I. Abdel-Aziz, H. M. Karara, and M. Hauck: Photogramm. Eng. Rem. S. 81 (2015) 103.

7 G. Xu, A. Zheng, X. Li, and J. Su: Sci. Rep. 6 (2016) 34591.

8 Z. Y. Zhang: IEEE Trans. Pattern Anal. 26 (2004) 892.

9 R. Tsai: IEEE Trans. Autom. Sci. Eng. 3 (1987) 323.

10 Z. Jia, J. Yang, W. Liu, F. Wang, Y. Liu, L. Wang, C. Fan, and K. Zhao: Opt. Express 23 (2015) 15205.

11 J. Santolaria, J. J. Aguilar, D. Guillomía, and C. Cajal: Robot CIM-INT Manuf. 27 (2011) 282.

12 H. Zhao, J. P. Kruth, N. Van Gestel, B. Boeckmans, and P. Bleys: Measurement 45 (2012) 1057.

13 M. Rahayem, N. Werghi, and J. Kjellander: Opt. Laser. Eng. 50 (2012) 1242.

14 J. E. Ha: Int. J. Control Autom. 10 (2012) 1240.

15 L. Ma, L. Wang, T. Cao, J. Wang, X. He, and C. Xiong: Meas. Sci. Technol. 18 (2007) 1768.

16 A. Fischer, C. Kupsch, J. Gürtler, and J. Czarske: Opt. Express 23 (2015) 24910.

17 M. Aymerich, D. Nieto, and M. T. Floresarias: Opt. Express 23 (2015) 24369.

18 M. H. Chiu, B. Y. Shih, and C. W. Lai: Appl. Phys. Lett. 90 (2007) 021111. 
19 W. Chen, S. Chen, D. Luo, and G. Jiao: Rev. Sci. Instrum. 86 (2015) 013102.

20 G. Xu, X. Zhang, J. Su, X. Li, and Z. Zheng: Appl. Opt. 55 (2016) 2653.

21 G. Xu, Z. Hao, J. Su, X. Li, H. Liu, and L. Sun: Sci. Rep. 5 (2015) 11928.

22 R. Hartley and A. Zisserman: Multiple View Geometry in Computer Vision (Cambridge University, Cambridge, 2003).

23 Z. Zhang: IEEE Trans. Pattern. Anal. 22 (2000) 1330.

24 O. Faugeras and Q. T. Luong: The Geometry of Multiple Images (MIT, Cambridge, 2001).

25 J. Nocedal and S. Wright: Numerical Optimization (Springer, Berlin, 2006).

26 J. Law and R. Rennie: A Dictionary of Physics (Oxford University Press, Oxford, 2015). 